\title{
A
}

Acta HealthMedica

Acta HealthMedica (ISSN: 2414-6528)

http://www.ActaHealthMedica.com

Volume: 2, Issue: 1, January-March 2017, Pages: 138, DOI: http://dx.doi.org/10.19082/ah138

\section{APPLYING DECISION TREE FOR DETECTION OF A RISK FACTORS FOR TYPE 2 DIABETES: A POPULATION BASED STUDY}

\author{
Maryam Tayefi ${ }^{1,3}$, Habibollah Esmaeily ${ }^{2}$,Majid Ghayour-Mobarhan ${ }^{1,3}$,Ali Reza Amirabadi zadeh ${ }^{4}$
}

1: Biochemistry of Nutrition Research Center, School of Medicine, Mashhad University of Medical Sciences, Mashhad, Iran.

2: Department of Biostatistics, School of Health, Mashhad University of Medical Sciences, Mashhad, Iran.

3: Department of Modern Sciences and Technologies, School of Medicine, Mashhad University of Medical Sciences, Mashhad, Iran.

4: Biostatistics, Medical Toxicology and Drug Abuse Research Center (MTDRC), Birjand University of Medical Sciences

Correspondence:

Ali Reza Amirabadi Zadeh, Tel:+989105090341,E-mail: amirabadiza921@gmail.com

\section{TYPE OF ARTICLE: CONFERENCE ABSTRACT}

\begin{abstract}
Introduction: The aim of current study was to create a prediction model using a data mining approach and decision tree technique to identify low risk individuals for incidence of type 2 diabetes (T2DM), using the Mashhad Stroke and Heart Atherosclerotic Disorders (MASHAD) Study program.

Methods: A prediction model was developed using classification by the decision tree method on 9528 subjects recruited from the MASHAD database. Moreover, the receiver operating characteristic (ROC) curve was applied.

Results: The prevalence rate of T2DM was $14 \%$ in our population. For the decision tree model, the accuracy, sensitivity, and specificity value for identifying the related factors with T2DM were $78.7 \%, 61.2 \%$, and $83 \%$, respectively. In addition, the area under the ROC curve (AUC) value for recognizing the risk factors associated with T2DM was $68 \%$. The identified variables included family history of diabetes, triglycerides, systolic blood pressure, body mass index, hs-crp, education.

Conclusion: Our findings demonstrated that decision tree analysis, using routine demographic, clinical, and anthropometric and biochemical measurements, which combined with other risk score models, could create a simple strategy to predict individuals at low risk for type 2 diabetes in order to substantially decrease the number of subjects needed for screening and recognition of subjects at high risk.

KEYWORDS: Data mining, Decision tree, Type 2 diabetes
\end{abstract}

\section{Abstracts of First National Congress of Medical Informatics, Mashhad, Iran, February 2017}

(C) 2017 The Authors. This is an open access article under the terms of the Creative Commons Attribution-NonCommercialNoDerivs License, which permits use and distribution in any medium, provided the original work is properly cited, the use is non-commercial and no modifications or adaptations are made. 